EPJ Web of Conferences 41, 11005 (2013)

DOI: $10.1051 /$ epjconf/20134111005

(C) Owned by the authors, published by EDP Sciences, 2013

\title{
Pulse shaping of on-chip microresonator frequency combs: investigation of temporal coherence
}

\author{
F. Ferdous, ${ }^{1}$ H. Miao, ${ }^{2,3}$ D. E. Leaird, ${ }^{1}$ K. Srinivasan, ${ }^{2}$ L. Chen, ${ }^{2}$ V. Aksyuk $^{2}$ and A. M. Weiner ${ }^{1,4}$ \\ ${ }^{1}$ School of Electrical and Computer Engineering, Purdue University,465 Northwestern Avenue, West \\ Lafayette, IN 47907-2035, USA \\ ${ }^{2}$ Center for Nanoscale Science and Technology, National Institute of Standards and Technology, 100 \\ Bureau Dr, Gaithersburg, MD 20899, USA \\ ${ }^{3}$ Nanocenter, University of Maryland, College Park, MD 20742, USA \\ ${ }^{4}$ Birck Nanotechnology Center, Purdue University, 1205 West State Street, West Lafayette, Indiana \\ 47907, USA
}

\begin{abstract}
We use pulse shaping to investigate the temporal coherence of frequency combs generated in microresonators pumped by a strong $\mathrm{CW}$ laser. We observe that different groups of comb lines have different mutual coherence.
\end{abstract}

There has been significant recent interest in comb generation methods based on high quality factor (Q) microresonators due to simplicity, small size, and compatibility with batch fabrication at low cost [1-7]. Different routes to comb formation are studied theoretically and experimentally in [5-7]. Most studies emphasize the frequency uniformity of the comb; time domain studies are relatively limited $[5,6]$. Combs formed as a cascade of sidebands spaced by a single free spectral range (FSR) that spread from the pump (Type I comb) exhibit high coherence. However, combs where the initial sidebands are spaced by multiple FSRs that then fill in to give combs with single FSR (Type II combs) show reduced coherence Here we study the properties of Type II combs, by investigating the time domain behaviors of a few subfamilies of frequency lines selected by a pulse shaper. We observe different coherent properties for different groups of comb lines.

We use a silicon nitride ring resonator with $100-\mu \mathrm{m}$ outer radius, $2-\mu \mathrm{m}$ width and $550-\mathrm{nm}$ thickness for the frequency comb generation as shown in Fig. 1a. Light is coupled into/out of the resonator via an on-chip waveguide with $1 \mu \mathrm{m}$ width and $800 \mathrm{~nm}$ ring-waveguide gap. The two ends of the waveguide are inversely tapered to $100 \mathrm{~nm}$ for low loss waveguide-fiber coupling. Single mode optical fibers are placed in the $\mathrm{V}$-grooves as shown in Fig. 1d and aligned with the waveguides, with coupling losses per facet of $\approx 1.5 \mathrm{~dB}$ achieved for lensed fibers. The intrinsic $\mathrm{Q}$ of the resonator is estimated to be $3.0 \times 10^{6}$. The average free spectral range (FSR) for the series of high-Q modes is measured to be $\approx 1.85 \mathrm{~nm}$.

Strong CW pumping light (estimated to be $27.6 \mathrm{dBm}$ into the accessing waveguide) is launched into an optical mode at around $1551.6 \mathrm{~nm}$. The generated frequency comb is sent to a line-by-line pulse shaper, which both assists in spectral phase characterization and enables pulse compression [6]. By slowly tuning the wavelength of the pump light from blue to red side, we first observed an optical frequency comb with spectral lines spaced by 3 FSR; then the spectral lines in between fill in to form a comb with one FSR spacing. Fig. $1 \mathrm{~b}$ and $1 \mathrm{c}$ show the spectrum of the 3 FSR spacing comb after equalization by the pulse shaper and the corresponding autocorrelation traces before and after phase

This is an Open Access article distributed under the terms of the Creative Commons Attribution License 2.0, which permits unrestricted use, distribution, and reproduction in any medium, provided the original work is properly cited. 
correction. In Fig 1c, the blue and red lines are the autocorrelation traces before and after phase correction, while the black line is the calculated autocorrelation trace for the spectrum shown in Fig $1 \mathrm{~b}$ with flat spectral phase. The measured autocorrelation trace after phase correction matches well with the calculated one, indicating high coherence. Fig $1 \mathrm{e}$ and $1 \mathrm{f}$ show the spectrum and the autocorrelation traces of the corresponding 1 FSR comb. The larger difference of the measured autocorrelation trace from the calculated one (in particular, the loss of autocorrelation contrast) indicates reduced coherence for this Type II comb.
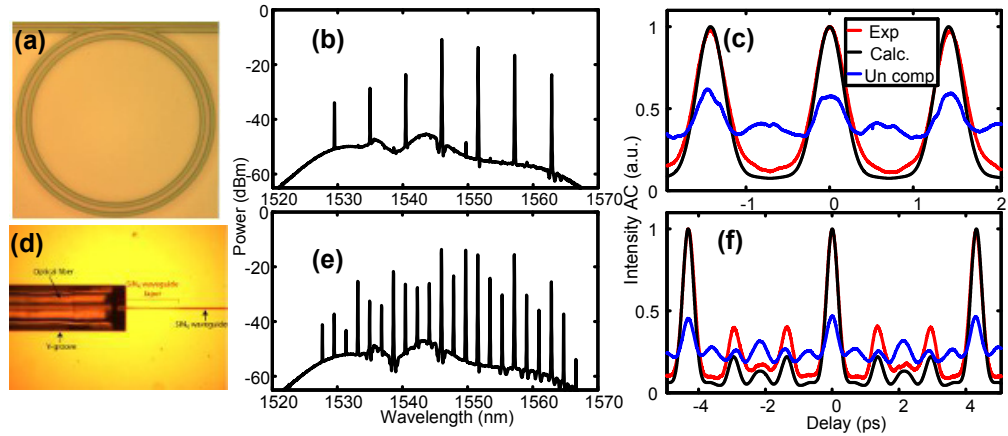

Fig.1. (a) Microscope image of a silicon nitride micro-ring with coupling region. (b) Spectrum of the generated 3 FSR spacing comb after the pulse shaper. (c) Autocorrelation traces corresponding to (b). (d) Image of coupling region in the V-groove. (e) Spectrum of the generated 1 FSR spacing comb after the pulse shaper. Here we tune the $\mathrm{CW}$ laser $53 \mathrm{pm}$ towards the red side than that of (b). (f) Autocorrelation traces corresponding to $(\mathrm{e})$.

To better understand the reduced coherence of a Type II comb, we use a pulse shaper to select subfamilies of comb lines from the spectrum shown in Fig. 1e, with 3 FSR comb spacing each, while keeping the initial phase profile the same as in the previous phase correction experiments in Fig. 1f. These selected spectra and corresponding autocorrelation traces are shown in Fig 2. Fig 2a and 2b show the result of the family of the comb lines corresponding to Fig $1 \mathrm{~b}$, for which the pump laser coincides with one of the lines. The very close agreement between experimental and ideal autocorrelations shows that high coherence is maintained for this subfamily of lines. Fig $2 \mathrm{c}-\mathrm{d}$ show the results of another family of 3 FSR lines shifted from the pump. The autocorrelation background and the mismatch between calculated and experimental autocorrelations increase significantly. These data show that different groups of lines in a Type II comb may have different coherence.

In another experiment, we explore the coherence of groups of three lines. The pulse shaper selects three lines from the comb of Fig. 1e and varies the relative phase $\Delta \Phi$ of the shortest wavelength line, while setting the phases of the other two lines to zero. As in [5], we observe the transition between constructive and destructive interference as we vary $\Delta \Phi$. Figure 3 shows the results. The left column shows the autocorrelation traces where $\Delta \Phi$ is zero (red) and $\pi$ (blue), the right column shows the visibility data. In both cases simulated results corresponding to full coherence are shown in black. A group of lines including the pump frequency (top row) shows strong dependence on $\Delta \Phi$, hence high temporal coherence; while a group of 3 lines shifted from the pump (bottom row) show little dependence on $\Delta \Phi$, hence low coherence. 

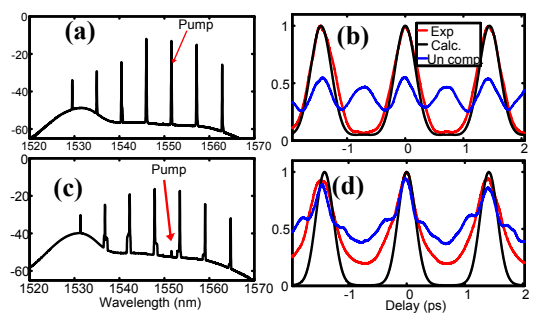

Fig.2. Spectra and autocorrelation traces for 2 subfamilies of comb lines selected from the spectrum shown in Fig. 1e.

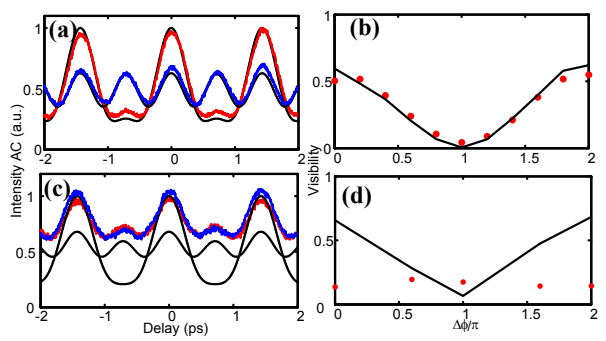

Fig.3. Autocorrelation traces (a,c) and visibility curves $(b, d)$ for 3 line experiments. In the intensity autocorrelations traces, results for $\Delta \Phi=0$ (red) and $\pi$ (blue) are plotted together with calculated results assuming full temporal coherence (black).

Our data reveal a striking variation in the degree of mutual coherence exhibited for different groups of lines selected out of the full, partially coherent comb. The structure observed in this and other measurements [8] of the mutual coherence provide evidence consistent with a simple model of partially coherent comb formation based on imperfect frequency division.

\section{References}

1. P. DelHaye, et al. Nature, 450, 1214 (2007)

2. J.S. Levy, et al. Nature Photonics, 4, 37 (2010)

3. L. Razzari, et al. Nature Photonics, 4, 41 (2010)

4. I.S. Grudinin, N. Yu, L. Maleki, Opt. Lett., 34, 878 (2009)

5. S.B. Papp, S.A. Diddams, Phy. Rev. A 84, 053833 (2011)

6. F. Ferdous, et al. Nature Photonics, 5, 770 (2011)

7. T. Herr, et al. Nature Photonics, 6, 480 (2012)

8. F. Ferdous, et al. http://arxiv.org/ftp/arxiv/papers/1207/1207.2134.pdf 